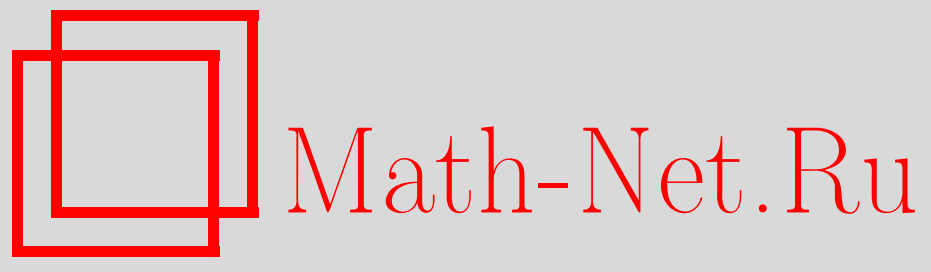

М. С. Мартиросян, С. В. Самарчян, О поперечниках Колмогорова множеств из экспонент, Матем. заметки, 2008, том 83, выпуск 2, 310-311

DOI: https://doi.org/10.4213/mzm4421

Использование Общероссийского математического портала Math-Net.Ru подразумевает, что вы прочитали и согласны с пользовательским соглашением http://www . mathnet.ru/rus/agreement

Параметры загрузки:

IP : 34.227 .88 .159

26 апреля 2023 г., 18:35:29

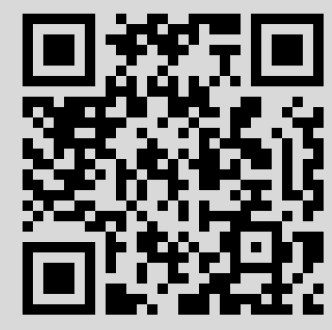




\section{О поперечниках Колмогорова множеств из экспонент}

\section{М. С. Мартиросян, С. В. Самарчян}

Пусть $K$ - компактное подмножество $\Pi_{+}=\{z \in \mathbb{C}: \operatorname{Re} z>0\}, S_{K}=\left\{e^{-\lambda x}: \lambda \in K\right\} \subset$ $L^{2}(0, \infty)$ и $d_{n}\left(S_{K}\right)-n$-поперечник Колмогорова множества $S_{K}$ в пространстве $L^{2}(0, \infty)$.

Тогда (см. [1])

$$
\limsup _{n \rightarrow \infty} \sqrt[n]{d_{n}\left(S_{K}\right)}<1
$$

для любого компакта $K \subset \Pi_{+}$.

Данная заметка посвящена следующему результату.

ТеоремА. Для выполнения условия

$$
\lim _{n \rightarrow \infty} \sqrt[n]{d_{n}\left(S_{K}\right)}=0
$$

необходимо и достаточно, чтобы логарифмическая емкость множества $K$ равнялась нулю.

ДоказАтельство. Пусть $\left\{\lambda_{k}\right\}_{1}^{\infty}$ - некоторое счетное подмножество $K$, а $E_{n}(f)$ - расстояние функции $f \in L^{2}(0, \infty)$ от подпространства, порожденное конечной системой $\left\{e^{-\lambda_{k} x}\right\}_{1}^{n}$. Если $f \in S_{K}$, то $E_{n}(f)$ удобно вычислить методом Мусояна (см. [1; с. 858]), что приводит к формуле

$$
E_{n}\left(e^{-\lambda x}\right)=\frac{1}{2 \pi} \frac{\left|\left(\lambda-\lambda_{1}\right)\left(\lambda-\lambda_{2}\right) \cdots\left(\lambda-\lambda_{n}\right)\right|}{\sqrt{2 \operatorname{Re} \lambda}\left|\left(\lambda+\bar{\lambda}_{1}\right)\left(\lambda+\bar{\lambda}_{2}\right) \cdots\left(\lambda+\bar{\lambda}_{n}\right)\right|}, \quad \operatorname{Re} \lambda>0 .
$$

Из этой формулы вытекает достаточность теоремы. Действительно, если $K$ имеет нулевую логарифмическую емкость, то в силу теоремы Фекете-Сегё (см. [2; с. 606-607]) существует треугольная матрица $\left(\lambda_{k}^{(n)}\right)_{k \leqslant n}, n=1,2, \ldots$, точек из $K$ такая, что

$$
\lim _{n \rightarrow \infty} \sqrt[n]{\sup _{\lambda \in K}\left|\left(\lambda-\lambda_{1}^{(n)}\right) \cdots\left(\lambda-\lambda_{n}^{(n)}\right)\right|}=0 .
$$

Для доказательства необходимости допустим обратное: существуют компакт $K \subset \Pi_{+}$ положительной логарифмической емкости и матрица $\left(e_{k}^{(n)}\right)_{k \leqslant n} \subset L^{2}(0, \infty)$ такие, что

$$
\rho_{n}=\sqrt[n]{\sup _{\lambda \in K}\left\|e^{-\lambda x}-\sum_{k=1}^{n} a_{k}^{(n)}(\lambda) e_{k}^{(n)}\right\|_{L^{2}(0, \infty)}} \underset{n \rightarrow \infty}{\longrightarrow} 0
$$

для некоторого набора коэффицентов $a_{k}^{(n)}(\lambda) \in \mathbb{C}, k \leqslant n$.

Пусть $\left(\lambda_{k}^{(n)}\right)_{k \leqslant n}-$ произвольная матрица точек из $K$. Так как соответствующая система векторов

$$
\left\{\left(a_{1}^{(n)}\left(\lambda_{i}^{(n+1)}\right), \ldots, a_{n}^{(n)}\left(\lambda_{i}^{(n+1)}\right)\right)\right\}_{i=1}^{n+1}
$$

линейно зависима в $\mathbb{C}^{n}$, то существуют коэффициенты $c_{i}^{(n+1)}, i=1, \ldots, n+1$, такие, что $\max _{1 \leqslant i \leqslant n+1}\left|c_{i}^{(n+1)}\right|=1$ и

$$
\sum_{i=1}^{n+1} c_{i}^{(n+1)} a_{k}^{(n)}\left(\lambda_{i}^{(n+1)}\right)=0, \quad k=1, \ldots, n .
$$

(C) М. С. МартиросЯн, С. В. СамарчЯн, 2008 
Рассмотрим полиномы

$$
P_{n+1}(x)=\sum_{i=1}^{n+1} c_{i}^{(n+1)} e^{-\lambda_{i}^{(n+1)} x}=\sum_{i=1}^{n+1} c_{i}^{(n+1)}\left(e^{-\lambda_{i}^{(n+1)} x}-\sum_{k=1}^{n} a_{k}^{(n)}\left(\lambda_{i}^{(n+1)}\right) e_{k}^{(n)}\right) .
$$

Согласно (2) имеем

$$
\lim _{n \rightarrow \infty} \sqrt[n]{\left\|P_{n+1}\right\|_{L^{2}(0, \infty)}} \leqslant \lim _{n \rightarrow \infty} \sqrt[n]{(n+1) \rho_{n}^{n}}=0
$$

С другой стороны, учитывая формулу (1), получаем

$$
\left\|P_{n+1}\right\|_{L^{2}(0, \infty)} \geqslant \frac{1}{2 \pi} \min _{1 \leqslant j \leqslant n+1} \frac{1}{\sqrt{2 \operatorname{Re} \lambda_{j}}} \prod_{i=1, i \neq j}^{n+1}\left|\frac{\lambda_{j}^{(n+1)}-\lambda_{i}^{(n+1)}}{\lambda_{j}^{(n+1)}+\bar{\lambda}_{i}^{(n+1)}}\right|
$$

и, следовательно,

$$
\liminf _{n \rightarrow \infty} \sqrt[n]{\left\|P_{n+1}\right\|_{L^{2}(0, \infty)}} \geqslant c \cdot \liminf _{n \rightarrow \infty} \min _{1 \leqslant j \leqslant n+1} \sqrt[n]{\prod_{i=1, i \neq j}^{n+1}\left|\lambda_{j}^{(n+1)}-\lambda_{i}^{(n+1)}\right|},
$$

где $c$ - положительная постоянная, не зависящая от набора точек $\lambda_{k}^{(n)}$.

Наконец, учитывая теорему Фекете-Сегё, точки $\lambda_{k}^{(n)}$ можно подобрать так, чтобы правая часть последнего неравенства оказалась положительной. Это противоречит оценке (3).

Теорема доказана.

\section{СПИСОК ЦИТИРОВАННОЙ ЛИТЕРАТУРЫ}

[1] B. X. Мусоян, Матем. заметки, 36:6 (1984), 857-863. [2] G. G. Lorentz, M. V. Golitschek, Y. Makovoz, Constructive Approximation. Advanced Problems, Grundlehren Math. Wiss., 304, Springer-Verlag, Berlin, 1996.

\section{М. С. Мартиросян}

Ереванский государственный университет

E-mail: approximation@gmail.com

\section{С. В. Самарчян}

Ереванский государственный университет

E-mail: approximation@gmail.com 